Original Paper http://ajol.info/index.php/ijbcs http://indexmedicus.afro.who.int

\title{
Caractérisation pathogénique, sérologique et moléculaire du virus de la mosaïque du niébé (Vigna unguiculata (L.) Walp.) transmis par pucerons (CABMV) dans les isolats du Burkina Faso, du Cameroun et de la Centrafrique
}

\author{
James B. NEYA ${ }^{1 *}$, Elisabeth P. ZIDA ${ }^{1}$, Innocent ZINGA ${ }^{2}$, Prosper ZEMBA ${ }^{1}$ et \\ Oumar TRAORE ${ }^{1}$
}

\author{
${ }^{1}$ Institut de l'Environnement et de recherches Agricoles (IN.E.R.A.), 04 P.O. Box 8645 Ouagadougou 04, \\ Burkina Faso. \\ ${ }^{2}$ Laboratoire des Sciences Biologiques et Agronomiques pour le Développement (LaSBAD), Département des \\ Sciences de la Vie, Université de Bangui, BP 908 Bangui, RCA. \\ *Auteur correspondant ; E-mail : neyajames@yahoo.fr ; Tel (226) 25319202 /08 ; Fax (226) 25319275
}

\section{RESUME}

Le niébé (Vigna unguiculata) est la principale légumineuse produite en Afrique. Sa production est confrontée à une contrainte majeure qui est la maladie de la mosaique du niébé transmise par pucerons. Cette maladie peut entraîner des pertes de rendement de 87\%. Les objectifs de cette étude étaient d'étudier les caractères sérologiques, moléculaires et la virulence du virus (CABMV) responsable de cette maladie au Burkina Faso, au Cameroun et en Centrafrique. Pour cela, la détection du virus par le test ACPELISA, l'inoculation mécanique, l'extraction de l'ARN viral total au TRizol et la RT-PCR ont été les méthodes utilisées pour caractériser le CABMV. Les caractérisations sérologique et moléculaire ont permis de détecter le CABMV sur 5 isolats et ont permis de confirmer l'existence du virus dans les trois pays. Plusieurs infections mixtes CABMV- CPMoV ont aussi été détectées. En outre, le criblage de dix variétés du Burkina Faso avec les cinq isolats a permis de distinguer quatre pathogroupes et un délai d'apparition de symptômes variant entre 6 et 20 JAI. Les variétés TZ1 Gourgou, KVx404-8-1x 693-2 BC4F9 et KVx 396-4-5-2Dx 693-2BC4F9 ont été résistantes à l'isolat RCA1. Les isolats BF et CAM2 ont été les plus virulents, suivis de RCA7, puis RCA4 et enfin RCA1.

(C) 2019 International Formulae Group. All rights reserved.

Mots clés: CABMV, CPMV, Mosaïques, Burkina Faso, Cameroun, Centrafrique.

\section{Pathogenic, serological and molecular characterization of cowpea (Vigna unguiculata (L.) Walp.) aphid borne mosaic virus (CABMV) in isolates from Burkina Faso, Cameroon and Central African Republic}

\begin{abstract}
Cowpea (Vigna unguiculata) is the main legume produced in Africa. Its production is facing to a major constraint, the mosaic disease transmitted by aphids. This disease may cause up to $87 \%$ loss. The objectives of
\end{abstract}


this study were to investigate the serological and molecular characteristics and virulence of virus (Cowpea Aphid Borne Virus (CABMV)) responsible of this disease in Burkina Faso, Cameroon and Central African Republic. For this purpose, the detection of the virus by ACP-ELISA, mechanical inoculation, the extraction of the total viral RNA by Trizol and RT-PCR were the methods used to characterize the CABMV. Serological and molecular characterization allowed detecting the CABMV in five isolates. This study confirmed the presence of the virus in the three countries. Several mixed infections of CABMV-CPMV were also detected. In addition, the screening of ten varieties from Burkina Faso with the five isolates allows identifying of four pathogroups and an appearance of symptoms period varying between 6 and 20 days after inoculation. TZ1 Gourgou, KVx 404-8-1x 693-2 BC4F9 and KVx 396-4-5[2Dx 693-2] BC4F9 were resistant to the isolate RCA1. The isolates $\mathrm{BF}$ and CAM2 were the most virulent, followed by RCA7 and RCA4 and finally RCA1.

(C) 2019 International Formulae Group. All rights reserved.

Keywords: CABMV, CPMV, Mosaic, Burkina Faso, Cameroon, Central African Republic.

\section{INTRODUCTION}

Le niébé (Vigna unguiculata (L.) Walp.) est une légumineuse alimentaire largement cultivée dans le monde avec une production annuelle estimée à 5,72 millions de tonnes de graines sèches dont $85 \%$ sont produites en Afrique (FAOSTAT, 2016). Cette légumineuse peut être cultivée dans la zone tropicale et subtropicale et s'adapte à différents types de sol. Les graines de niébé sont riches en acides aminés et fournissent 20 à $25 \%$ de protéines et $64 \%$ de carbohydrates pour les populations (Adigoun, 2002; Akubor, 2003 ; Jackson, 2009 ; Modu et al., 2010). Un des aspects les plus importants de l'apport du niébé dans l'alimentation humaine est la teneur de ses protéines en acides aminés essentiels comme la lysine, le tryptophane, la phénylalanine, la valine, la thréonine, la méthionine, etc. (USDA, 2004). Ainsi, le niébé joue un rôle important dans l'équilibre nutritionnel des populations du Sahel dont l'alimentation est largement à base de céréales (Ouédraogo, 2003 ; Atachi et al., 2002). Selon Niang (2004), le niébé est consommé par près de 200 millions de personnes en Afrique tropicale. De ce fait le niébé est une plante de choix surtout dans les pays en développement. Elle est capable de fixer l'azote atmosphérique grâce aux bactéries du genre rhizobium qui se trouvent dans les nodosités de ses racines et peut fixer jusqu'à 240 kg/ha (Bado, 2002; Kaboré, 2004 ; Husson et al., 2010).

$\mathrm{Au}$ regard de ces potentialités, le Burkina Faso s'est donc doté dès 2003 d'un plan d'action spécifique pour le développement de la filière niébé (MAHRH, 2003). La mise en œuvre de cette politique a permis d'atteindre en 2014 une production de 599804 tonnes (MASA, 2014). Le revenu issu de la vente du niébé estimé aujourd'hui à 20 milliards de francs CFA par an représente 2,1\% du revenu monétaire agricole burkinabé (Dabat et al., 2012).

Malgré l'augmentation de la production et des superficies induites par cette politique, le niébé reste moins performant en rendements qui stagnent autour de $700 \mathrm{~kg} / \mathrm{ha}$ (MASA, 2014). Cette faiblesse est engendrée par de nombreuses contraintes abiotiques et biotiques dont les plus importantes sont : la pauvreté des sols, la mauvaise répartition des pluies, l'action de nombreux ravageurs et maladies provoquant d'importantes pertes de récolte.

Parmi les maladies affectant la production du niébé, les maladies virales ont une forte incidence économique et sont difficiles à combattre. Cinq maladies virales affectant la production du niébé ont été identifiées au Burkina Faso dont les plus importantes sont : la maladie de la marbrure du niébé (CPMoV), la maladie de la mosaïque du haricot commun (BlCMV) et la maladie de la mosaïque du niébé propagée par les pucerons (CABMV) qui est la plus répandue.

Néya (2011) a montré que la mosaïque du niébé propagée par pucerons (Aphis craccivora, A. gossypii, A. fabae) est la plus importante maladie virale du niébé au Burkina Faso au regard de sa distribution 
géographique et de son incidence économique. Selon Néya et al. (2015), la propagation de cette maladie peut se faire à partir des parcelles voisines infectées. La maladie de la mosaïque du niébé a pour agent pathogène le Cowpea aphid-borne mosaic virus (CABMV) ou virus de mosaïque du niébé transmis par les pucerons. Il appartient à la famille des Potyviridae qui comprend six genres (Fauquet et al., 2005). Le genre Potyvirus est le plus important groupe dans la famille des Potyviridae avec une centaine de membres (Fauquet et al., 2005). Ces membres ont des particules virales à structure filamenteuse (Sharma et al., 2013).

Les pertes de récolte engendrées par ce virus sont comprises entre 15 et $87 \%$ selon les variétés de niébé et l'âge des plants au moment de l'infection. Kareem et Taiwo (2007) ont obtenu des pertes totales de récolte chez trois variétés sensibles de niébé (IT86D719 , Olo-II et Olloyin) lorsque les plants étaient infectés à l'âge de 10 à 30 jours. L'évaluation de 21 variétés de niébé vis-à-vis de ce virus au Burkina Faso a conduit à des pertes situées entre 3 à $67 \%$ (Néya et al., 2015).

De nombreuses études ont permis de proposer des méthodes de lutte qui n'ont pas pu résoudre de façon durable les problèmes causés par le CABMV. Au Burkina Faso, Néya (2011) a proposé une stratégie de gestion intégrée du CABMV basée sur l'usage de variétés résistantes associé à des traitements chimiques réalisés à des dates bien précises. Cependant la mise en œuvre de cette stratégie passe d'abord par la recherche de génotypes résistants et l'approfondissement des connaissances sur le virus. En effet, les aspects moléculaires du virus restent à étudier et les aspects pathogéniques ont besoin d'être affinés. La transmission des virus par graines est une propriété dont les mécanismes sont complexes (De Assis Filho et Sherwood, 2000 ; Ng et Perry, 2004). De plus, aucun traitement n'est disponible pour éliminer le virus dans les graines infectées sans endommager leur pouvoir germinatif (Bashir et al., 2002). Le CABMV est transmissible par les graines de niébé à des taux allant de 3 à
$100 \%$ selon la variété (Barro et al., 2016). Cette propriété est la plus importante source d'infection primaire responsable de l'apparition des premiers plants infectés au champ.

L'objectif général de cette étude était de déterminer les caractéristiques sérologiques, pathogéniques, moléculaires et phylogéniques du virus de la mosaïque du niébé transmis par pucerons dans des échantillons du Burkina Faso, de la Centrafrique et du Cameroun. L'objectif spécifique était d'étudier la virulence des isolats provenant de ces trois pays dans le cadre d'une collaboration entre pays du Sud pour la mise au point de variétés dotées d'une résistance stable à la maladie.

\section{MATERIEL ET METHODES}

\section{Sources d'inoculum}

Les graines de niébé étant la source d'inoculum primaire du CABMV, 11 variétés provenant du Burkina Faso, du Cameroun, et de la République Centrafricaine ont été utilisées pour la recherche du CABMV (Tableau 1). Deux cents graines de chaque variété ont été traitées au Calthio $\mathrm{C}$ à la dose de $4 \mathrm{~g} / \mathrm{kg}$ de semences. Ces graines ont été ensuite semées séparément dans des bacs à l'abri des insectes sous une serre. La terre des bacs a été constamment maintenue humide en arrosant à la demande. Les plants de chaque variété comportant des symptômes (mosaïques, marbrures) ont été identifiés par observation visuelle du $7^{\text {ème }}$ au $14^{\text {ème }}$ jour après semis (JAS). Aussi, au $14^{\text {ème }} \mathrm{JAS}$, de l'engrais NPK (14-23-14) a été épandu à la dose de $100 \mathrm{~kg} / \mathrm{ha}$, soit $0,5 \mathrm{~kg}$ par bac.

\section{Technique de transmission par inoculation mécanique}

L'inoculum provenant de jeunes plants de chaque variété a été homogénéisé dans du tampon phosphate de sodium 0,01 M, pH 7,4 selon un rapport de broyage de $1 / 10(\mathrm{p} / \mathrm{v})$. Avant l'inoculation, les feuilles de jeunes plants sains de ces variétés âgés de deux semaines ont été nettoyées avec du coton et saupoudrées avec du carborundum 600 mesh. A l'aide d'un bâtonnet ouaté ou du pilon 
trempé dans l'extrait, la surface supérieure des feuilles a été frottée délicatement.

\section{Anticorps utilisés}

Deux anticorps polyclonaux spécifiques au CABMV et au CPMoV ont été utilisés pour la détection des virus dans les échantillons. L'anticorps poly clonal $\mathrm{n}^{\circ} 1$ est dirigé contre le Cowpea aphid-borne mosaic virus (CABMV), le $\mathrm{n}^{\circ} 2$ contre le Cowpea mottle virus (CPMoV).

\section{Détection sérologique des virus dans les feuilles par ACP-ELISA}

Le test Enzyme Linked ImmunoSorbent Assay (ELISA) variance Antigen-Coated Plate (ACP) a été utilisé pour la détection des virus selon le protocole suivant :

Broyage des échantillons dans du tampon coating plus $1 \%$ de sodium bisulfite dans un rapport de $0,3 \mathrm{~g}$ pour $4 \mathrm{~mL}$.

Distribution des extraits de broyats filtrés à raison de $100 \mu \mathrm{L}$ par puits et incubation pendant $2 \mathrm{~h}$ à $37^{\circ} \mathrm{C}$ suivie d'une saturation avec du lait écrémé 3\% dilué dans du Tampon Phosphate Salin Tween (PBS-T) et incubation pendant $30 \min$ à $37^{\circ} \mathrm{C}$.

Incubation des anticorps anti CABMV et anti CPMoV dilués à $1 / 5000$ dans du tampon PBST plus $1 \%$ de lait écrémé et $2 \%$ de PVP 2 h à $37{ }^{\circ} \mathrm{C}$ ou toute la nuit à $4{ }^{\circ} \mathrm{C}$.

Incubation du conjugué Goat AntiRabbit (GAR) couplé à la phosphatase alcaline (PAL) diluée à 1/5000 dans du tampon PBST plus $1 \%$ de lait écrémé à $37^{\circ} \mathrm{C}$ à raison de $100 \mu \mathrm{L} /$ puits pendant $2 \mathrm{~h}$.

Incubation du substrat Para-nitrophényl phosphate (pNPP) dissous dans le tampon substrat $(\mathrm{pH} \mathrm{9,8)}$ à une concentration de $1 \mathrm{mg} / \mathrm{mL}$ et distribué dans les plaques (100 $\mu \mathrm{L} /$ puits) pendant $1 \mathrm{~h}$ à $37^{\circ} \mathrm{C}$. Au terme du temps d'incubation, le lecteur de plaque Metertech $\Sigma 960$ a été utilisé pour lire chaque puits à la densité optique à $405 \mathrm{~nm}$.

Les plaques ont été rincées trois fois après chaque étape excepté celle de la saturation.

\section{Etude de la diversité pathogénique du CABMV}

Des plants sains de dix variétés de niébé (Tableau 2) ont été produits dans des pots. Les échantillons détectés positifs au CABMV par ACP-ELISA ont été inoculés mécaniquement à 10 jeunes plants de chaque variété. Une grille d'observation a été utilisée pour la collecte des données. Le nombre de plants infectés a été enregistré chaque jour entre les $6^{\text {ème }}$ et $21^{\text {ème }}$ jours après inoculation.

Les différents types de symptômes et leurs sévérités ont également été enregistrés. A la fin des observations, les feuilles des plants inoculés ayant donné des symptômes ou non ont été testées par ACP-ELISA pour confirmer la présence ou non du virus inoculé.

\section{Caractérisation moléculaire du CABMV par RT-PCR}

\section{Extraction de l'ARN au TRIzol}

Les mortiers et les pilons ont été préalablement stérilisés à $150{ }^{\circ} \mathrm{C}$ pendant $2 \mathrm{~h}$ ou toute la nuit, puis placés à $-80^{\circ} \mathrm{C}$ en même temps que les échantillons. Les feuilles froides de niébé ont été broyées et reprises dans un tube eppendorf froid de $2 \mathrm{~mL}$ dans lequel on y a ajouté $1000 \mu \mathrm{L}$ de TRIzol (stocké à $4{ }^{\circ} \mathrm{C}$ ) et $200 \mu \mathrm{L}$ de chloroforme froid avant d'homogénéiser. Les tubes ont été maintenus dans la glace pendant $5 \mathrm{~min}$. Une centrifugation à $13000 \mathrm{rpm}$ pendant $15 \mathrm{~min}$ à $4{ }^{\circ} \mathrm{C}$ a été faite afin de séparer la phase aqueuse. Le surnageant a été transféré dans un nouveau tube stérile de $1,5 \mathrm{~mL}$, auquel $550 \mu \mathrm{L}$ d'isopropanol froid ont été ajoutés et les tubes ont été placés à $-20{ }^{\circ} \mathrm{C}$ environ 30 min. Le mélange a ensuite été centrifugé à $13000 \mathrm{rpm}$ pendant $10 \mathrm{~min}$ à $4{ }^{\circ} \mathrm{C}$. Le culot ainsi obtenu a été lavé dans $500 \mu \mathrm{L}$ d'éthanol froid $75 \%$ par centrifugation à $12000 \mathrm{rpm}$ pendant 5 min toujours à $4{ }^{\circ} \mathrm{C}$. Après séchage, le culot a été repris dans $30 \mu \mathrm{L}$ d'eau distillée stérile. Un traitement à la Dnase a suivi l'extraction des ARN au Trizol.

\section{Traitement des ARN à la Dnase}

La Dnase a pour but de détruire les ADN résiduels contenus dans les échantillons. A cet effet, $10 \mu \mathrm{g}$ d'ARN ont été mélangés à 
$2 \mu \mathrm{L}$ de tampon Dnase 10X (Euromedex) et à $10 \mu \mathrm{L}$ de Dnase $(1 \mathrm{U} / \mu \mathrm{L})$ Euromedex. Ce mélange a été complété à $20 \mu \mathrm{L}$ avec de l'eau distillée stérile puis incubé à $37^{\circ} \mathrm{C}$ pendant 30 min. $1 \mu \mathrm{L}$ d'EDTA $25 \mathrm{mM}$ a été ajouté au mélange suivie d'une deuxième incubation effectuée à $65{ }^{\circ} \mathrm{C}$ pendant $10 \mathrm{~min}$. Les ARN ainsi obtenus ont été analysés quantitativement et qualitativement.

\section{Analyse quantitative et qualitative des ARN extraits}

Un dosage au spectrophotomètre a été effectué afin de s'assurer de la qualité des ARN extraits. Les ARN ont été dilués 80 fois ; soit $5 \mu \mathrm{L}$ d'ARN dans $395 \mu \mathrm{L}$ d'eau distillée stérile avant de mesurer la densité optique (DO) à $260 \mathrm{~nm}$ et $280 \mathrm{~nm}$. Le rapport de la DO à $260 \mathrm{~nm}$ sur la DO à $280 \mathrm{~nm}$ a été calculé afin d'apprécier la qualité des différents extraits. Les concentrations ont pu être calculées, car une absorbance d'une unité correspond à une concentration de $40 \mu \mathrm{g} / \mathrm{mL}$ d'ARN simple brin.

Les ARN ainsi obtenus ont été vérifiés par électrophorèse sur un gel d'agarose à $1 \%$. A cet effet, $1 \mathrm{~g}$ d'agarose a été dissout dans $100 \mathrm{ml}$ de TAE $1 \mathrm{X}$ (Tris-Acétate-EDTA). Ce mélange a été chauffé au four micro-onde jusqu'à ébullition, puis $1 \mu \mathrm{L}$ de Bromure d'éthidium (BET) a été ajouté après un refroidissement à environ $60^{\circ} \mathrm{C}$. Ce mélange a été coulé et une fois le gel solidifié, il a été placé dans la cuve d'électrophorèse contenant du TAE 1X. Les puits ont été chargés avec $5 \mu \mathrm{l}$ d'ARN. Le gel a été soumis à une tension de 100 volts et les produits chargés ont migré pendant $10 \mathrm{~min}$. A la fin de la migration, le gel a été observé sur une table à Ultra-Violets (UV) afin de vérifier la qualité des ARN extraits.

\section{Technique de Reverse Transcription (RT)}

Les ARN obtenus devaient être rétro transcrits en ADNc (ADN complémentaire) avant d'être amplifiés. Pour ce faire, $1 \mu \mathrm{g}$ d'ARN a été ajouté à $1 \mu \mathrm{L}$ d'oligo dT $(10 \mu \mathrm{M})$ et le volume a été complété à $5 \mu \mathrm{L}$ avec de l'eau distillée stérile. Ce mélange a été incubé à $70{ }^{\circ} \mathrm{C}$ pendant $10 \mathrm{~min}$ afin de dénaturer les ARN qui y sont contenus. Après incubation, les tubes ont été plongés immédiatement dans la glace pendant $10 \mathrm{~min}$.

Ensuite, il a été ajouté à la solution précédente $1 \mu$ Lde Tampon MMLV (Moloney Murine Leukemia Virus) Promega, $5 \mu \mathrm{L}$ de tampon RT Promega, $1,25 \mu \mathrm{L}$ de dNTP (10 $\mu \mathrm{M})$ et $0,5 \mu \mathrm{L}$ de Rnase inibitor. Le volume total a été complété à $25 \mu \mathrm{L}$ avec de l'eau distillée stérile. Les mélanges ont été incubés à $42{ }^{\circ} \mathrm{C}$ pendant $60 \mathrm{~min}$. L'ADNc (ADN complémentaire) ainsi obtenu a été conservé à $-20{ }^{\circ} \mathrm{C}$ pour l'étape de la réaction de polymérase en chaîne (PCR).

Technique de Réaction de Polymérase en Chaîne (PCR)

Après la transcription inverse, un thermocycleur DNA engine PTC-200 a été utilisé pour l'amplification des $\mathrm{ADNc}$ obtenus. Pour ce faire, $5 \mu \mathrm{L}$ de l'ADNc ont été ajoutés à $1 \mu \mathrm{L}$ de l'amorce sens (Oligo $1 \mathrm{n}$ ) Eurogentec spécifique à la protéine de la capside à $10 \mu \mathrm{M}$ (ATGGTHTGGTGYATHGARAAYGG), puis $1 \mu \mathrm{L}$ de l'amorce antisens (Oligo 2n) (TGCTGCKGCYTTCATYTG) à la même concentration. Le volume final a été complété à $25 \mu \mathrm{L}$ avec $12,42 \mu \mathrm{L}$ d'eau distillée stérile. Les tubes ont été incubés dans un thermocycleur suivant le programme: Une dénaturation à $95{ }^{\circ} \mathrm{C}$ pendant $5 \mathrm{~min}$, suivie de 35 cycles de 1 min à $94{ }^{\circ} \mathrm{C}$, puis $55^{\circ} \mathrm{C}$ durant $1 \mathrm{~min}$, et $72{ }^{\circ} \mathrm{C}$ pendant $1 \mathrm{~min}$. Le programme s'achève par une étape d'élongation finale à $72{ }^{\circ} \mathrm{C}$ pendant $10 \mathrm{~min}$.

A la fin de l'amplification, une électrophorèse de $12 \mu \mathrm{L}$ de chaque produit PCR a été effectuée sur du gel d'agarose (1\%) durant 20 min à 100 volts dans du TAE. A cet effet, $1 \mathrm{~kb}$ DNA ladder a été utilisé comme marqueur de taille. Une photo du gel a été prise sur une table à Ultra-violet. Chacun des 5 produits RT-PCR issu des isolats CABMV a été complété à $32 \mu \mathrm{L}$ avec de l'eau distillée stérile puis le tout a été envoyé pour le séquençage.

\section{Analyse des données}

Le tableur Excel 2007 a été utilisé pour les calculs et la construction des différentes figures. 
Tableau1: Sources d'inoculum.

\begin{tabular}{lllll}
\hline $\mathbf{N}^{\mathbf{0}}$ & Variétés & Code & Origine & Pays de collecte \\
\hline 1 & Nord Cameroun & CAM1 & Ngaoundéré & Cameroun \\
2 & Ouest Cameroun & CAM2 & Cbaf & Cameroun \\
3 & Gorom local & BF & Gorom-Gorom & Burkina Faso \\
4 & Haricot blanc & RCA1 & Kapou 2 & République centrafricaine \\
5 & TVX 19490F & RCA2 & Kaga Bandoro & République centrafricaine \\
6 & Petevo (petits grains lisses) & RCA3 & Kabo & République centrafricaine \\
7 & $\begin{array}{l}\text { Petevo (petits grains à œil } \\
\text { blanc) }\end{array}$ & RCA4 & Paoua & République centrafricaine \\
8 & $\begin{array}{l}\text { Petevo (gros grains) } \\
9\end{array}$ & RCA5 & Kaga Bandoro & République centrafricaine \\
10 & $\begin{array}{l}\text { Petevo (petits grains à } \text { œeil } \\
\text { noir) }\end{array}$ & RCA7 & Paoua & République centrafricaine \\
11 & Tchad & RCA8 & Tchad & République centrafricaine \\
\hline
\end{tabular}

Tableau 2: Variétés criblées.

\begin{tabular}{llcl}
\hline $\mathbf{N}^{\mathbf{0}}$ & Variétés & Localités & Pays d'origine \\
\hline 1 & TZ 1 Gourgou & Tenkodogo & Burkina Faso \\
2 & TS Donsin & Donsin & Burkina Faso \\
3 & Gorom local & Gorom-Gorom & Burkina Faso \\
4 & KVx 61-1 & Kamboinsé & Burkina Faso \\
5 & KVx771-10 G & Kamboinsé & Burkina Faso \\
6 & KVx745-11P & Kamboinsé & Burkina Faso \\
7 & KVx775-33-2 G & Kamboinsé & Burkina Faso \\
8 & KVx396-4-5-2D x 693-2BC4F9 & Kamboinsé & Burkina Faso \\
9 & KVx404-8-1 x 693-2 BC4F9 & Kamboinsé & Burkina Faso \\
10 & KVx775-33-2 x 693-2 BC4F9 & Kamboinsé & Burkina Faso \\
\hline
\end{tabular}




\section{RESULTATS \\ La sérologie}

Les tests sérologiques ACP-ELISA dont les résultats sont reportés dans le Tableau 3 ont permis de détecter le Cowpea aphidborne mosaic virus (CABMV) dans 5 échantillons, et le Cowpea mottle virus (CPMoV) dans 7 échantillons.

Le seuil de détection du CABMV était de 0,392 contre $0,531 \mathrm{chez}$ le CPMoV. Il a été calculé comme étant la moyenne des DO à $405 \mathrm{~nm}$ obtenues pour les témoins sains, augmentée de trois fois leur écart-type. Plusieurs co-infections virales (CABMV, $\mathrm{CPMoV}$ ) ont été relevées par le diagnostic sur deux variétés de Centrafrique, une variété du Cameroun et une variété du Burkina Faso.

\section{La pathogénie}

Les 5 isolats testés positifs au CABMV ont été inoculés mécaniquement à 10 variétés de niébé provenant du Burkina Faso. Les résultats des interactions niébé- CABMV ont révélé plusieurs types de symptômes ainsi que différents délais d'apparition des symptômes variant de 6 à 21 jours après inoculation (JAI).

De façon générale, les symptômes observés lors de la caractérisation pathogénique sont des mosaïques jaunes ou vertes. Celles-ci peuvent être accompagnées de déformations foliaires et de chutes de feuilles en cas de sévères infections. Plusieurs types de symptômes sont souvent observés sur le même plant. Le Tableau 4 et la Figure 1 récapitulent les divers symptômes observés sur les 10 variétés criblées.

\section{Interactions entre l'isolat RCA1 et les variétés testées}

Les résultats obtenus après l'inoculation de l'isolat RCA1 aux plants sains des dix variétés testées sont illustrés par la Figure 2. On constate que l'isolat RCA1 a induit les premiers symptômes 6 JAI chez la moitié des variétés testées. La variété Gorom local (sensible de référence) a exprimé ses premiers symptômes 14 JAI. Chez la variété KVx775-33-2G, les premiers symptômes ont été observés entre le $18^{\text {ème }}$ et le $20^{\text {ème }}$ JAI. Les variétés KVx771-10G, TS Donsin et Gorom local ont enregistré $100 \%$ de plants infectés contre $80 \%$ chez la variété $\mathrm{KVx} 61-1$. Les variétés $\quad \mathrm{KV} \times 775-33-2-\mathrm{x} 639-2 \quad \mathrm{BC} 4 \mathrm{~F} 9$, KVx775-33-2G et KVx745-11P ont eu respectivement $37,5 \% ; 50 \%$ et $55,56 \%$ de plants infectés après inoculation de RCA1. Les variétés $\mathrm{KVx} 404-8-1 \times$ 693-2 BC4F9 et KVx396-4-5-2Dx 693-2 BC4F9 et TZ 1 Gourgou n'ont pas exprimé de symptômes jusqu'à la fin des observations qui ont duré un mois.

\section{Interactions entre l'isolat RCA4 et les variétés testées}

Les résultats obtenus après l'inoculation de l'isolat RCA4 aux plants sains des dix variétés testées sont illustrés par la Figure 3. Ces résultats font remarquer que l'isolat RCA4 a induit des symptômes chez toutes les dix variétés. TZ Gourgou, KVx3964-5-2Dx693-2BC4F9 et Gorom local ont été les plus infectées avec $100 \%$ de plants malades tandis que les variétés $\mathrm{KVx} 404-8$ 1x693-2, KVx775-33-2x639-2 BC4F9 et $\mathrm{KV} 745-11 \mathrm{P}$ ont été moins infectées avec $22 \%$ et $33 \%$ de plants infectés. La variété sensible de référence (Gorom local) a atteint les $100 \%$ de plants infectés au $14^{\text {ème }}$ JAI.

Interactions entre 1'isolat RCA7 et les variétés testées

L'inoculation de l'isolat RCA7 aux plants sains des dix variétés a donné les résultats représentés par la Figure 4. Cette figure montre que l'isolat RCA7 a aussi induit des symptômes entre 6 et 20 JAI. Les variétés les moins infectées KVx775-33-2x6392BC4F9 et KVx396-4-5-2Dx693-2 BC4F9 ont enregistré $50 \%$ de plants malades tandis que les variétés $\mathrm{KVx} 404-8-1 \times 693-2 \mathrm{BC} 4 \mathrm{~F} 9$, $\mathrm{KV} \times 775-33-2 \mathrm{G}$ et $\mathrm{KV} \times 61-1$ et Gorom local ont été les plus infectées avec $100 \%$ de plants malades.

\section{Interactions entre l'isolat CAM2 et les variétés testées}

Les résultats obtenus après l'inoculation de l'isolat CAM2 aux plants sains des dix variétés testées sont illustrés par la Figure 5. Ils indiquent que l'isolat CAM2 a infecté plus de $50 \%$ des plants de toutes les variétés au bout de 20 JAI. Les variétés TZ1 Gourgou (résistante), KVx775-33-2x693- 
2BC4F9, KVx396-4-5-2Dx693-2BC4F9, KVx775-33-2G, Gorom local (sensible) et TS Donsin ont enregistré 100\% de plants malades. La variété $\mathrm{KVx} 771-10 \mathrm{G}$ a été la moins infectée avec 50\% de plants qui ont exprimé des symptômes.

\section{Interactions entre l'isolat BF et les variétés testées}

Les résultats obtenus après l'inoculation de l'isolat BF aux plants sains des dix variétés testées sont représentés par la Figure 6. On constate que l'isolat BF a infecté plus de $60 \%$ des plants de toutes les variétés criblées. Les délais d'apparition des symptômes ont varié de 6 à 20 JAI. Six variétés ont jusqu'à $100 \%$ de plants infectés. Il s'agit de KVx775-33-2G, KVx396-4-5-2Dx693-2BC4F9, KVx771-10G, Gorom local, KVx61-1 et TS Donsin. L'isolat BF a été plus virulent que les autres.

\section{Détection sérologique du CABMV dans les plants inoculés}

Les résultats des tests sérologiques réalisés à l'issu de la caractérisation pathogénique sont résumés dans le Tableau 5. Ils montrent que les isolats RCA4, RCA7, CAM2 et BF ont infecté toutes les 10 variétés testées. Quant à l'isolat RCA1, il n'a pas pu infecter les variétés TZ1 Gourgou, KVx404-81x693-2BC4F9 et KVx396-4-5-2-Dx 6932BC4F9. Les feuilles des plants sans symptômes ont répondu négativement au test ELISA.

\section{Caractérisation moléculaire}

Après l'extraction des ARN totaux, un dosage au spectrophotomètre et une migration sur gel d'agarose ont été effectués pour s'assurer de la quantité et de la qualité des ARN totaux. L'extraction au TRIZOL a permis d'avoir des ARN de bonne qualité comme l'indique la Figure 7.

L'amplification du gène de la protéine de la capside du CABMV par RT-PCR a été un succès en utilisant un couple d'amorces dégénérées fourni par Eurogentec. Il a permis d'obtenir des amplicons de la CP à la taille de 327 paires de bases (Figure 8).

Tableau 3: Détection des différents virus dans les 11 échantillons.

\begin{tabular}{|c|c|c|c|}
\hline & & CABMV & CPMoV \\
\hline 0 & Témoin négatif & - & - \\
\hline $0^{\prime}$ & Témoin positif & + & + \\
\hline 1 & RCA 1 & + & - \\
\hline 2 & RCA 2 & - & - \\
\hline 3 & RCA 3 & - & + \\
\hline 4 & RCA 4 & + & + \\
\hline 5 & RCA 5 & - & - \\
\hline 6 & RCA 6 & - & + \\
\hline 7 & RCA 7 & + & + \\
\hline 8 & RCA 8 & - & - \\
\hline 9 & CAM 1 & - & + \\
\hline 10 & CAM 2 & + & + \\
\hline 11 & BF & + & + \\
\hline
\end{tabular}


Tableau 4 : Différents symptômes induits par isolat et par variété.

\begin{tabular}{|c|c|c|c|c|c|c|}
\hline No & Variétés/Isolats & RCA1 & RCA4 & RCA7 & CAM2 & BF \\
\hline 1 & TZ 1 Gourgou & - & MJ & MJ & MJ & MJ \\
\hline 2 & $\begin{array}{l}\text { KVx404-8-1x693- } \\
\text { 2BC4F9 }\end{array}$ & - & MJ & MJ & MV & $\mathrm{MJ}, \mathrm{MV}, \mathrm{DF}$ \\
\hline 3 & $\begin{array}{l}\text { KVx775-33-2x693- } \\
\text { 2BC4F9 }\end{array}$ & MJ & MJ & MJ & $\mathrm{MJ}, \mathrm{MV}, \mathrm{CF}$ & MJ \\
\hline 4 & $\begin{array}{l}\text { KVx396-4-5- 2Dx693- } \\
2 \text { BC4F9 }\end{array}$ & - & MJ & MJ & MJ, MV & MJ, MV \\
\hline 5 & $\mathrm{KV} \times 775-33-2 \mathrm{G}$ & MJ & MJ & MJ, DF & MJ & MJ, MV \\
\hline 6 & Gorom local & MJ & MJ & MJ, DF & MV & $\begin{array}{c}\text { MJ, MV, DF. } \\
\text { CF }\end{array}$ \\
\hline 7 & $\mathrm{KV} \times 745-11 \mathrm{P}$ & $\mathrm{MV}, \mathrm{DF}$ & MJ & MJ, MV, DF & MJ, MV & MJ, MV \\
\hline 8 & KVx771-10G & MJ & MJ, MV & MJ & MJ & MJ, MV \\
\hline 9 & TS Donsin & $\mathrm{MV}, \mathrm{DF}$ & MJ & MJ, MV & MJ & MJ, MV \\
\hline 10 & KVx61-1 & MJ & MJ, MV DF & MJ, MV & MJ & MJ, MV \\
\hline
\end{tabular}

Tableau 5: Vérification sérologique à l'issu du criblage.

\begin{tabular}{|c|c|c|c|c|c|c|}
\hline $\mathbf{N}^{\mathbf{0}}$ & Variétés & RCA1 & RCA4 & RCA7 & CAM2 & $\mathbf{B F}$ \\
\hline 1 & TZ1 Gourgou & - & + & + & + & + \\
\hline 2 & KVx404-8-1x 693-2 BC4F9 & - & + & + & + & + \\
\hline 3 & KVx775-33-2 x693-2 BC4F9 & + & + & + & + & + \\
\hline 4 & KVx396-4-5-2-Dx693-2BC4F9 & - & + & + & + & + \\
\hline 5 & $\mathrm{KV} \times 775-33-2 \mathrm{G}$ & + & + & + & + & + \\
\hline 6 & Gorom local & + & + & + & + & + \\
\hline 7 & $\mathrm{KV} \times 745-11 \mathrm{P}$ & + & + & + & + & + \\
\hline 8 & KVx771-10G & + & + & + & + & + \\
\hline 9 & TS Donsin & + & + & + & + & + \\
\hline 10 & KVx61-1 & + & + & + & + & + \\
\hline
\end{tabular}


B. J. NEYA et al. / Int. J. Biol. Chem. Sci. 13(1): 382-398, 2019

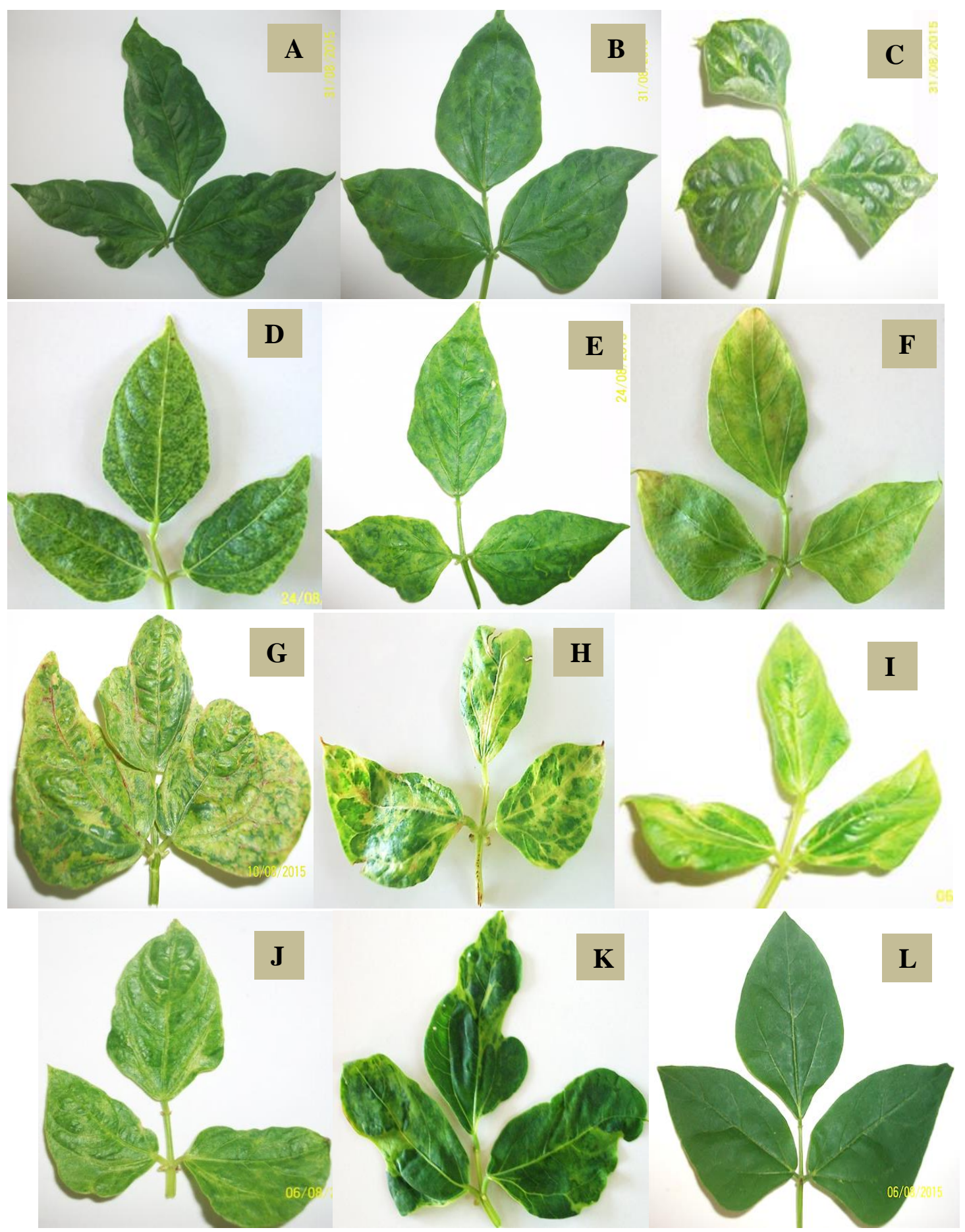

Mosaïque Verte (A, B, C) ; Mosaïque Jaune (D, E, F) ; Mosaïques sévères (G, H, I, J K), Témoin sain (L).

Figure1: Différents symptômes observés. 


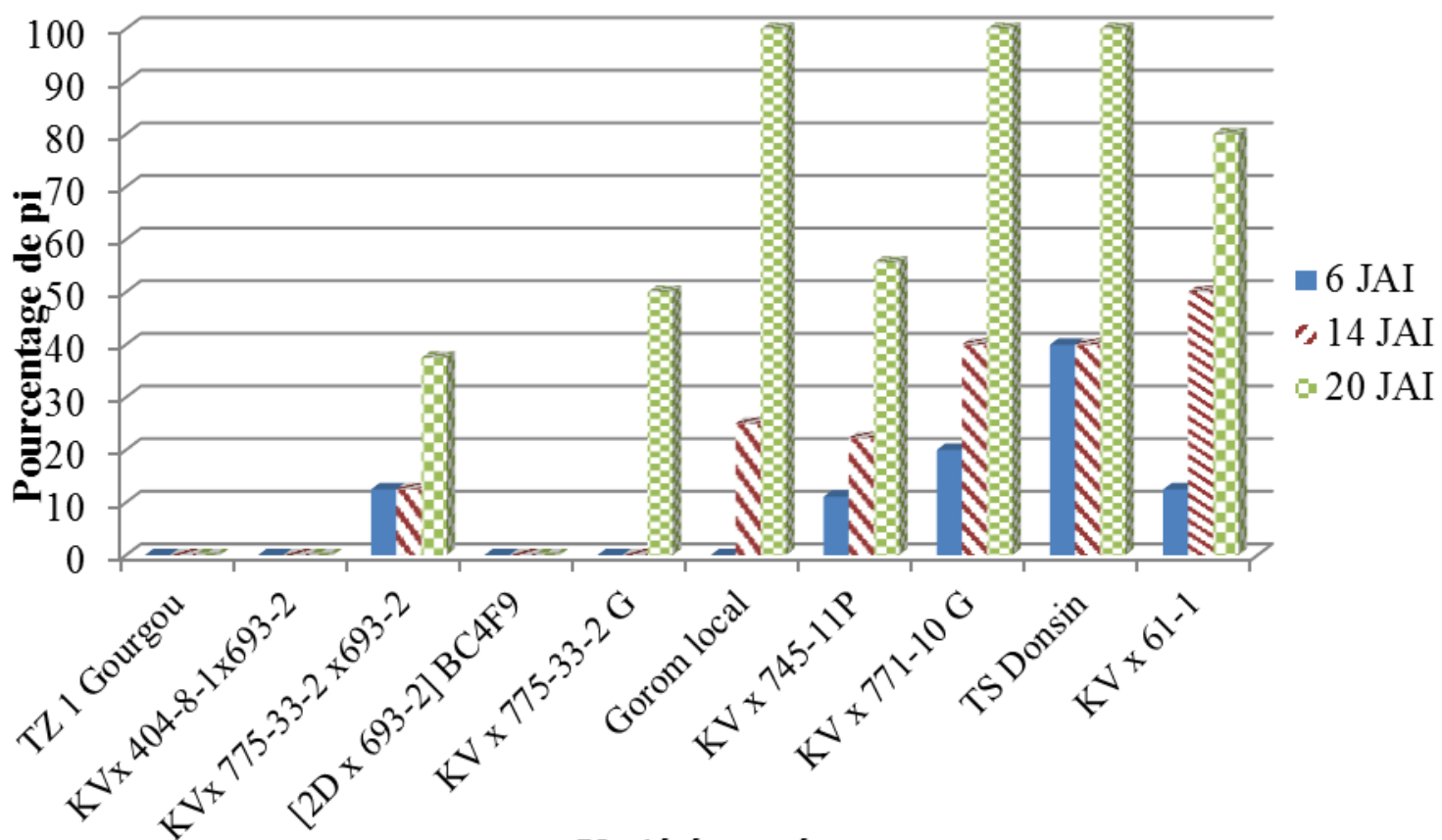

Variétés testées

Figure 1: Réactions des variétés testées avec l'isolat RCA1. $\mathrm{Pi}=$ plants infectés.

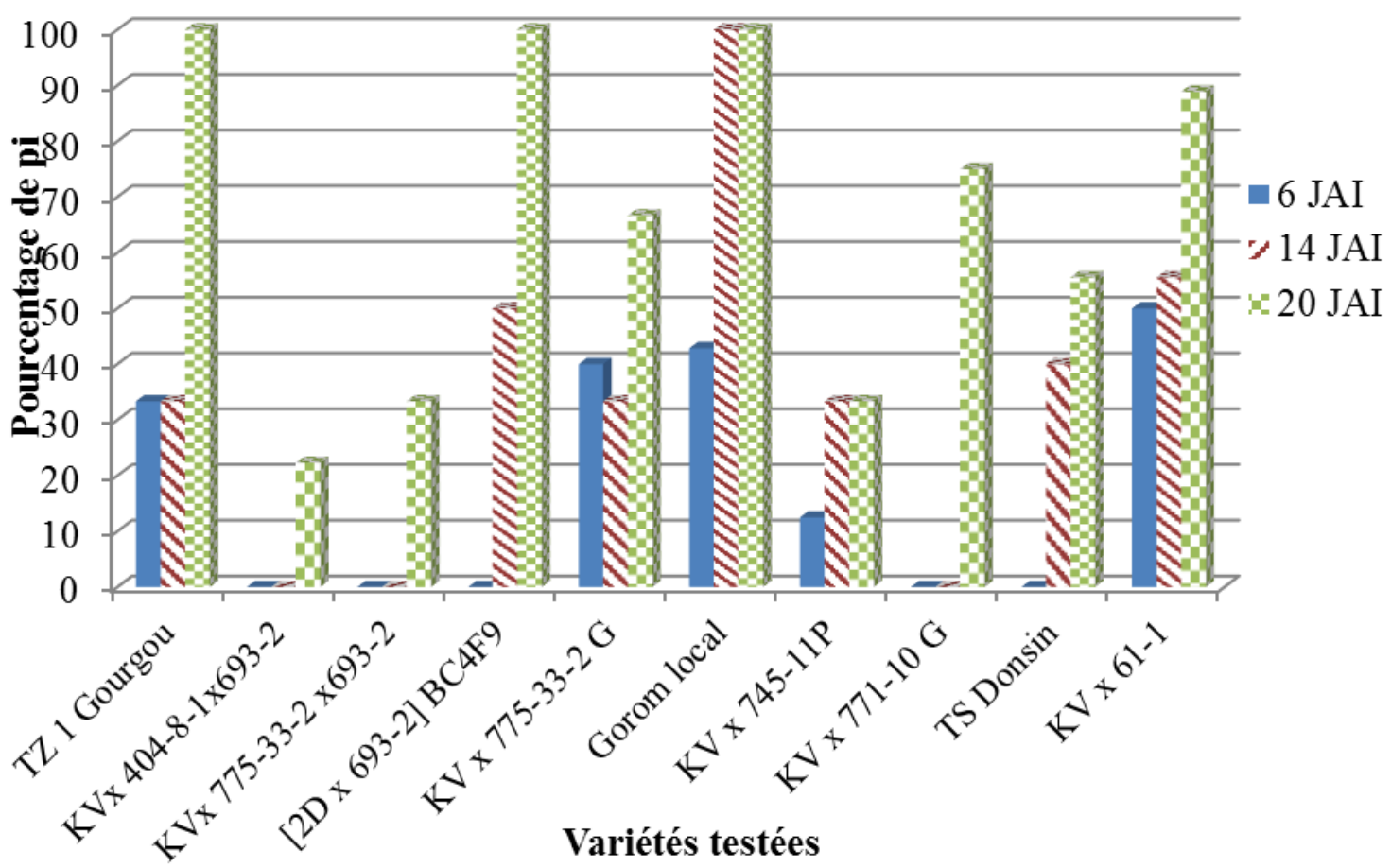

Figure 2: Réactions des variétés testées avec l'isolat RCA4.

$\mathrm{Pi}=$ plants infectés. 


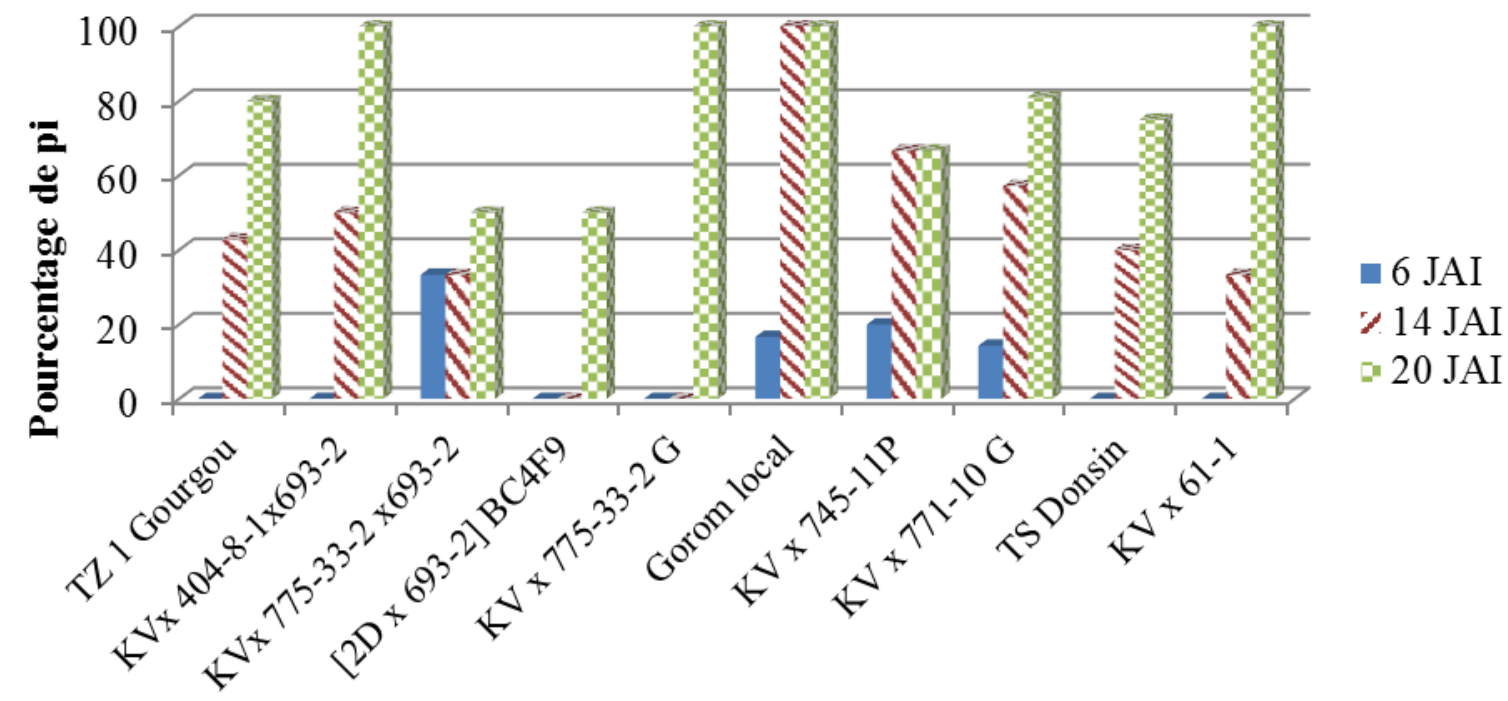

Variétés testées

Figure 3: Réactions des variétés testées avec l'isolat RCA7.

$\mathrm{Pi}=$ plants infectés.

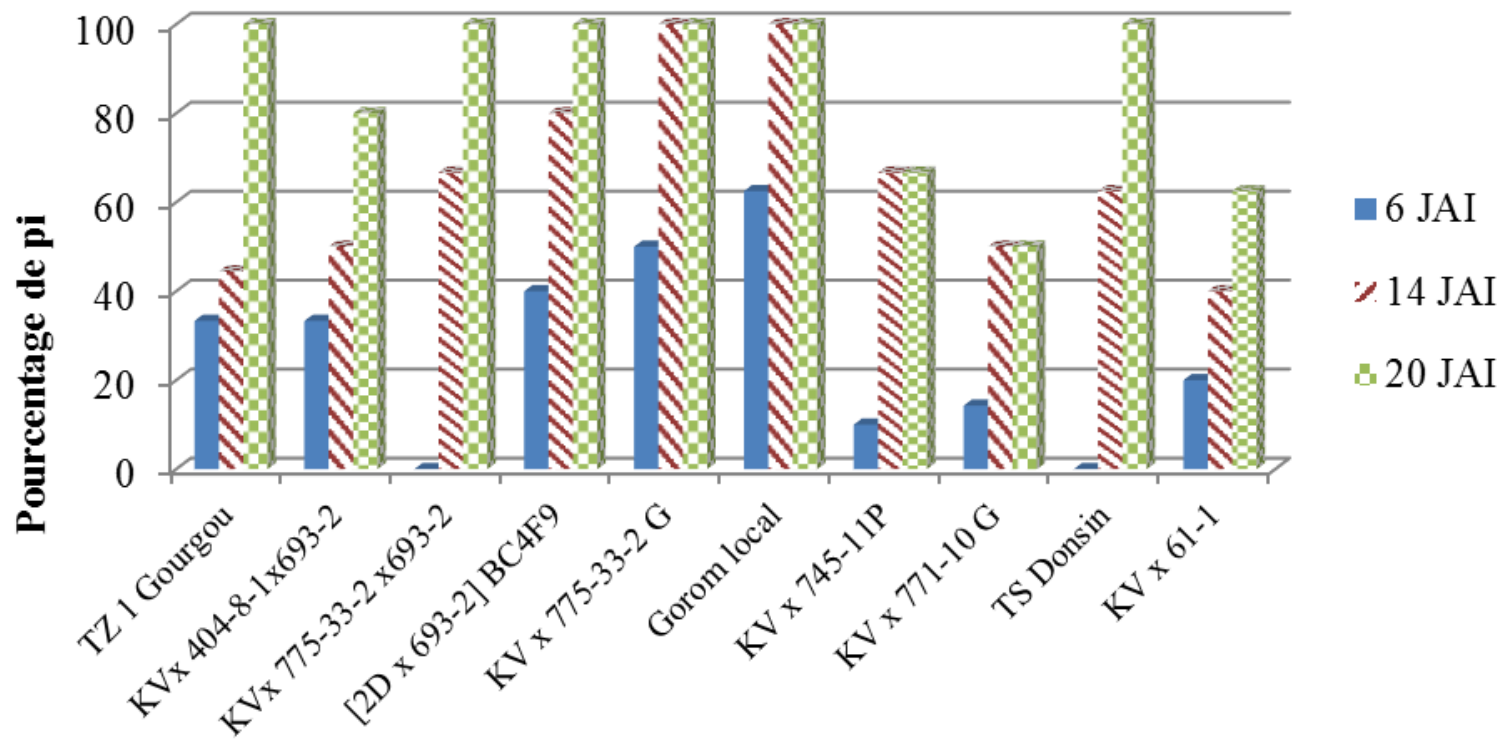

\section{Variétés testées}

Figure 4: Réactions des variétés testées avec l'isolat CAM2. $\mathrm{Pi}=$ plants infectés. 


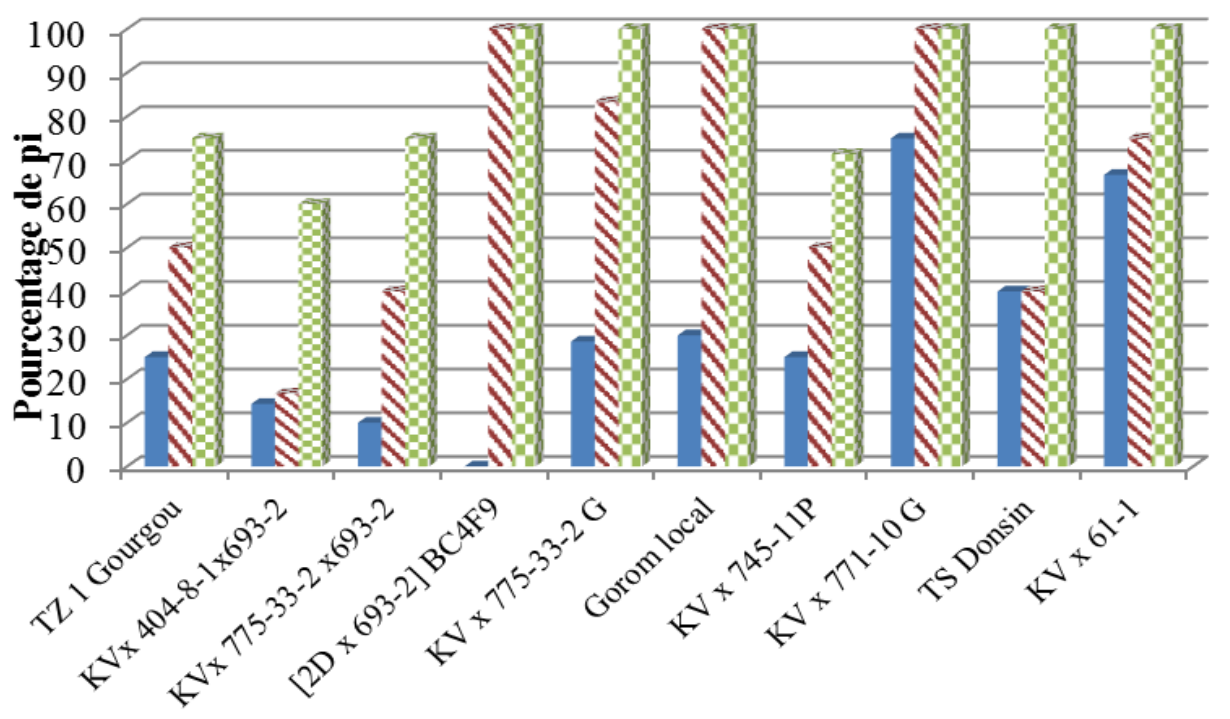

Figure 5: Réactions des variétés testées avec l'isolat BF. $\mathrm{Pi}=$ plants infectés.

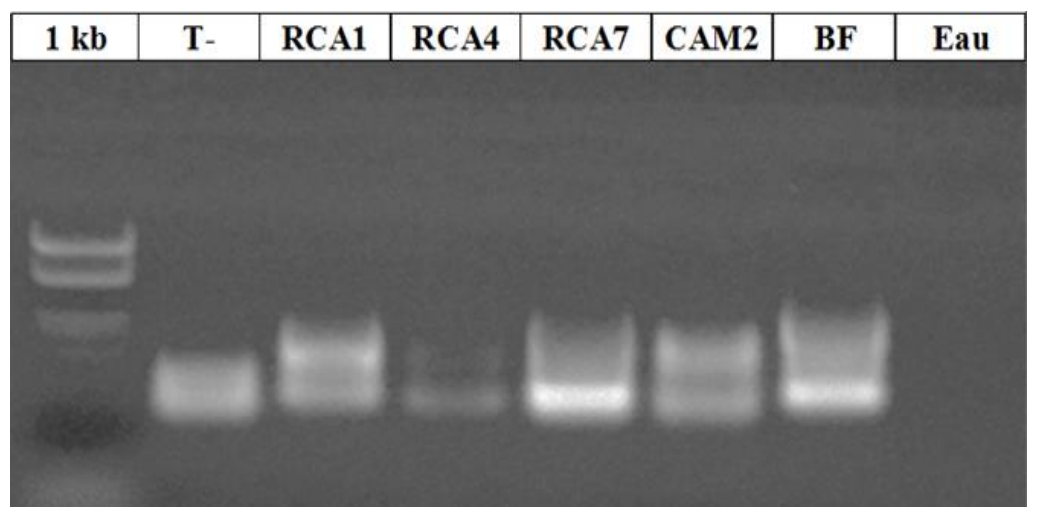

Figure 6: Vérification par électrophorèse des ARN sur gel d'agarose à $1 \%$.

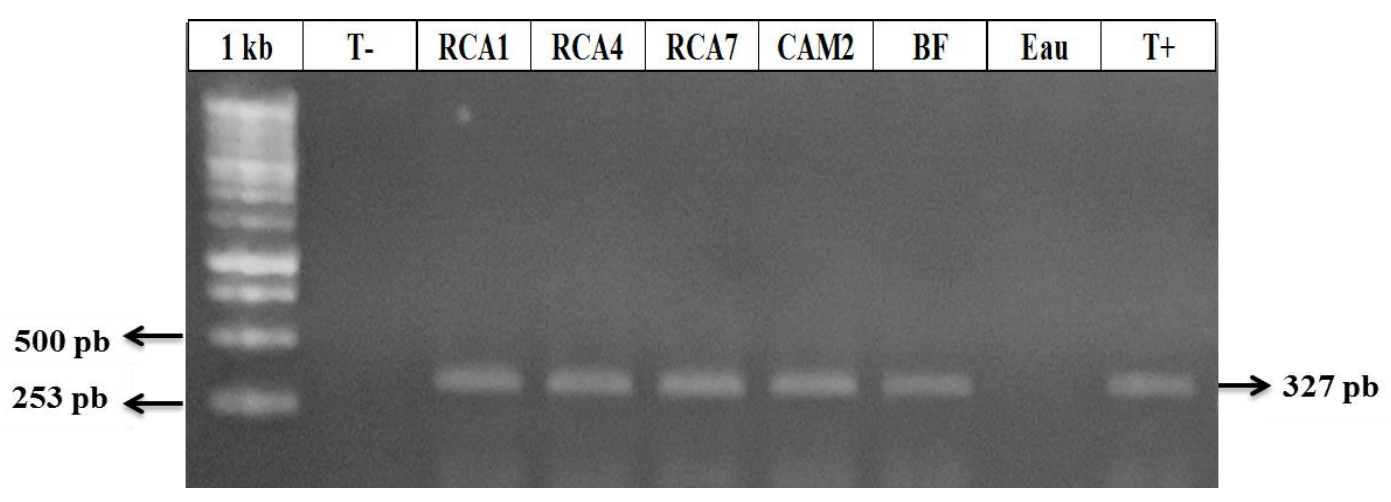

Figure 7: Electrophorèse des produits RT-PCR du gène de la CP des isolats. 


\section{DISCUSSION}

La sérologie: le test ACP-ELISA a permis de détecter le Cowpea aphid-borne mosaic virus (CABMV) au Burkina Faso, au Cameroun et en Centrafrique avec des niveaux de réaction (valeur d'absorbance A405 nm) très variables. $\mathrm{La}$ variation d'absorbance peut s'expliquer par des différences de concentration virale dans les échantillons. Elle peut aussi traduire une variabilité du CABMV entre échantillons. Ces résultats montrent que les différences d'absorbance reflètent des réactivités plus ou moins fortes entre les souches virales et les anticorps utilisés.

Les tests sérologiques, ont également permis de détecter le Cowpea mottle mosaic virus (CPoMV) en co-infection avec le CABMV dans des échantillons du Burkina Faso, du Cameroun et de la Centrafrique. La possibilité pour plusieurs virus du niébé d'être transmis par voie mécanique ou par un même groupe d'insectes est souvent à l'origine d'infections mixtes dans les plants. Orawu et al., (2015) avaient également révélé l'existence de nombreuses infections virales mixtes sur le niébé.

La pathogénie: l'inoculation mécanique des 5 isolats à 10 variétés de niébé du Burkina au stade 2 feuilles a induit diverses mosaïques généralisées. Cela prouve que le CABMV est bien transmissible mécaniquement et qu'il est systémique. Ces résultats confirment ceux de Hampton et Tottappilly (2003).

Les variétés n'ont pas réagi de la même manière à l'inoculation du même virus. En effet, les délais d'apparition des symptômes à l'issu des inoculations ont varié de 6 à $20 \mathrm{JAI}$. Ces résultats sont en accord avec ceux de Néya (2011) qui a affirmé que les premiers symptômes apparaissaient entre 6 et 7 JAI pour les variétés sensibles et qu'il fallait attendre 14 à 21 JAI pour observer des symptômes sur les variétés tolérantes.

L'isolat RCA1 n'a pas induit de symptômes sur les variétés TZ1 Gourgou, KVx404-8-1x 693-2BC4F9 et KVx396-4-5-2Dx 693-2BC4F9. Le test ACP-ELISA effectué sur les plants inoculés a permis de confirmer l'absence de l'isolat RCA1 dans ces trois variétés d'où leur résistance. Cependant, ces trois variétés résistantes à l'isolat RCA1 ont eu des réactions différentes avec les autres isolats viraux. L'isolat RCA7 a induit 100\% d'infection sur TZ1 Gourgou tandis que les isolats CAM2 et $\mathrm{BF}$ ont induit $100 \%$ d'infection respectivement sur $\mathrm{KVx} 404-8$ 1x693-2 BC4F9 et KVx 396-4-5-[2Dx 6932]BC4F9. Cette réaction opposée peut s'expliquer par le fait que la résistance du niébé qui est gouvernée par deux gènes est vite contournée par l'une des nombreuses souches du CABMV. Nos résultats confirment ceux de Bashir et al., (2002) et de Barro et al.,(2016) qui ont signalé que la variabilité entre isolats du CABMV influence fortement l'expression des symptômes et la capacité d'isolats du virus à infecter ou non certaines variétés de niébé.

Toutes les infections dues au CABMV étaient accompagnées de symptômes. Ainsi, quatre pathogroupes ont été déterminés à savoir des mosaïques vertes, des mosaïques jaunes, des mosaïques vertes avec déformation foliaire, des mosaïques jaunes avec déformation foliaire accompagnées par moment de chute de feuilles dans les cas d'infections sévères. Ces mosaïques sont semblables à celles décrites par Néya (2002) et Nédjim (2013).

La co-infection CABMV et CPMoV a été une contrainte dans l'étude pathogénique, car il existe une certaine interaction entre les virus ce qui pourrait avoir accéléré ou ralenti l'infection. Aussi, les mosaïques dues au CPMoV peuvent également induire des confusions de symptômes. Cela montre que deux virus différents peuvent induire des symptômes identiques. Les isolats $\mathrm{BF}$ et CAM2 ont été les plus virulents, suivis de RCA7, puis RCA4 et enfin RCA1.

La RT-PCR : les résultats des tests de biologie moléculaire concordent avec les données sérologiques et confirment la présence du CABMV au Burkina Faso, au Cameroun et en Centrafrique. En effet, les amplicons visualisés après migration des produits PCR sur gel d'agarose étaient tous à la bonne taille $(327 \mathrm{pb})$, prouvant qu'une 
portion de la protéine de la capside du CABMV a été bien amplifiée. Ces résultats sont similaires à ceux de Wulandari et Ermayanti (2011) qui avaient obtenu des amplicons de même taille en utilisant les mêmes amorces pour détecter le CABMV.

\section{Conclusion}

L'objectif de ce travail était d'étudier la virulence des isolats provenant de ces trois pays dans le cadre d'une collaboration entre pays du Sud pour la mise au point de variétés dotées d'une résistance stable à la maladie.

Les données collectées au cours de ce travail nous ont apporté de précieuses informations sur le Cowpea aphid-borne mosaic virus (CABMV) tant pour le Burkina Faso que pour le Cameroun et la Centrafrique.

D'abord, l'étude sérologique (ACPELISA) a permis la détection du CABMV dans des échantillons de niébé provenant de ces trois pays. Dans plusieurs cas le CABMV était en infection mixte avec le Cowpea mosaic virus $(\mathrm{CPMoV})$ toute chose qui a pu entraîner des confusions de symptômes. Une autre étude pourrait permettre d'appréhender la distribution géographique de ce virus en Centrafrique et les pertes de production qu'il pourrait occasionner.

Ensuite, l'étude pathogénique par inoculation mécanique des virus à des variétés du Burkina Faso a permis de distinguer quatre (4) pathogroupes à savoir des mosaïques vertes, des mosaïques jaunes, des mosaïques vertes avec déformation foliaire et des mosaïques jaunes avec déformation. Trois variétés résistantes à l'isolat RCA1 ont été déterminées (TZ1 Gourgou, KVx404-8-1x 693-2BC4F9 et KVx396-4-5-2Dx 6932BC4F9). Ces variétés pourraient être utilisées comme variétés différentielles pour différencier l'isolat RCA1 des deux autres isolats (RCA4 et RCA7) de la Centrafrique.

Enfin, l'étude moléculaire (RT-PCR) a permis de confirmer l'étude sérologique par l'amplification d'une portion du gène de la protéine de la capside du CABMV grâce à des amorces dégénérées.

\section{CONFLIT D'INTERETS}

Les auteurs déclarent qu'il $\mathrm{n}$ y a aucun conflit d'intérêt.

\section{CONTRIBUTIONS DES AUTEURS}

BJN a suivi des tests, collecté des données et a participé à la rédaction du manuscrit. PEZ a suivi les tests, collecté les données et a participé à la correction du manuscrit. ZI collecté les échantillons de RCA et a participé à la correction du manuscrit. ZP a suivi des tests, collecté des données et a participé à la rédaction du manuscrit. OT a supervisé les travaux, analysé les données et a corrigé le manuscrit.

\section{REMERCIEMENTS}

Nous remercions le LMI-Patho-Bios pour l'appui nécessaire aux analyses sérologiques. Nous sommes reconnaissants envers Martine Bangraz pour son assistance dans les analyses TR-PCR.

\section{REFERENCES}

Adigoun FA. 2002. Impact des traitements phytosanitaires du niébé sur l'environnement et la santé des populations : cas de Klouékanmé et de la basse vallée de l'Ouémé (Bénin). Mémoire de maitrise professionnelle, Université d'abomey calavi (uac), Cotonou, 71p.

Akubor PI. 2003. Functional properties and performance of cowpea/plantain/wheat flour blends in biscuits. J. Plant Food Hum. Nutr., 58: 1-8. DOI: 10.1023/B: QUAL. 0000040342.75650.5c

Atachi P, Dannon AE, Arodokoun DY, Tamo M. 2002. Distribution and sampling of Maruca vitrata (FABRICIUS) (Lep., Pyralidae) larve on Lonchocarpus sericeus (PÜIR) H B. and K. J. Appl. Ent., 126: 188 - 193. DOI: 10.1046/j.1439-0418.2002. 00633.x

Bado BV. 2002. Rôle des légumineuses sur la fertilité des sols ferrugineux tropicaux des zones guinéennes et soudanienne du Burkina Faso. Thèse de l'Université Laval, 197 p. 
Barro A, Sawadogo M, Kiébré Z, Néya BJ. 2016. Evaluation de la résistance de quelques lignées et écotypes de niébé (Vigna unguiculata (L.) WALP.) au Cowpea Aphid-Borne Mosaic Virus au Burkina Faso. Int. J. Inn. Appl. Stud., 15: 387-394.

http://www.ijias.issrjournals.org/

Bashir M, Hamad Z, Ghafoor A. 2002. Cowpea aphid-borne mosaic potyvirus: a review. Int. J. Pest Manag., 48: 155168. DOI:10.1080/09670870110118722.

Dabat M-H, Lahmar R, Guissou R. 2012. La culture du niébé au Burkina Faso: une voie d'adaptation de la petite agriculture à son environnement? Presses de Sciences Po., 62: 95-114. DOI: 10.3917/autr.062.0095

De Assis Filho FM, Sherwood JL. 2000. Evaluation of seed transmission of Turnip yellow mosaic virus and Tobacco mosaic virus in Arabidopsis thaliana. Phytopathology, 90: 1233-1238. DOI: 10.1094/PHYTO.2000.90.11.1233

FAO STAT. 2016. Production végétale, quantité produite et superficies emblavées. FAO STAT. http://faostat.fao.org.

Fauquet CM, Mayo MA, Maniloff J, Desselberger U, Ball LA. 2005. Virus taxonomy. Eighth Report of the International Committee on Taxonomy of Viruses. Elsevier Academic Press: San Diego; $1259 \mathrm{p}$.

Hampton RO, Thottappilly G. 2003. Cowpea. Virus and Virus-like Diseases of Major Crops in Developing Countries. In: Loebenstein G; Thottappilly G. (eds). Kluwer Academic Publishers; 355-376.

Husson O, Charpentier H, Razanamparany C, Moussa N, Michellon R, Naudin K, Razafintsalama H, Rakotoarinivo C, Rakotondramanana, Enjalric F, Seguy L. 2010. Maïs ou sorgho associé à une légumineuse alimentaire volubile (Dolique, Niébé ou Vigna umbellata). Manuel pratique du semis direct à Madagascar, Volume I : Chapitre I.

Jackson JC. 2009. Protein nutritional quality of cowpea and navy bean residue fractions. African J. Food Agric., Nutrition and Develop., 9: 764-778. DOI: http://dx.doi.org/10.4314/ajfa nd.v9i2.19225.

Kabore B. 2004. Les contributions en azote des légumineuses, des amendements organominéraux dans les systèmes de culture: impact sur les rendements des céréales et su la fertilité des sols à long terme. Mémoire IDRI/Agronomie/Université Polytechnique de Bobo dioulasso, Burkina Faso, 77 p.

Karem KT, Taïwo MA. 2007. Interaction of viruses in cowpea: effects on growth and yield parameters. Virol. J., 4 : 15-21. DOI: $\quad 10.1186 / 1743-422 X-4-$ 15.http://www.virologyj.com/content/4/1 $/ 15$.

MAHRH. 2003. Plan d'action pour le développement de la filière niébé au Burkina Faso, Ministère de l'Agriculture, de l'Hydraulique et des Ressources halieutiques (MAHRH), Secrétariat permanent de la coordination des politiques sectorielles agricoles (SP/CPSA), version finale. MAHRH, $114 \mathrm{p}$.

MASA. 2014. Résultats définitifs de la campagne agricole et de la situation alimentaire et nutritionnelle 2013/2014. MASA, 72p.

Modu Y, Putai AJ, Petu-Ibikunle AM. 2010. An Economic Analysis of Cowpea Production among Women Farmers in Askira/Uba Local Government Area Borno State Nigeria. Afr. J. General Agric., 6: 7-17. http://www.asopah.org

Nédjim V. 2013. Evaluation des pertes de rendement causées par le virus de la mosaïque du niébé (Vigna unguiculata (L.) Walp.) transmis par pucerons (CABMV). Mémoire de master professionnel en protection et amélioration des plantes, Université d'Ouagadougou, $54 \mathrm{p}$.

Néya BJ. 2002. Variabilité sérologique et aspects épidémiologiques du virus de la mosaïque du niébé (Vigna unguiculata (L.) Walp.) transmis par des pucerons au 
Burkina Faso. Mémoire de DEA en sciences biologiques appliquées, UFR/SVT, Université de Ouagadougou, Burkina Faso, 47p.

Néya BJ. 2011. Sérologie, pathogénie, épidémiologie et contrôle de la mosaïque Cowpea aphid-borne mosaic virus (CABMV) du niébé (Vigna unguiculata (L.) Walp.) transmis par pucerons par des pucerons (Aphis craccivora, A. gossypii) au Burkina Faso. Thèse unique, Université de Ouagadougou, 153p.

Néya BJ, Zida PE, Tignégré JBS, Traoré O. 2015. Etude de l'effet d'une source d'inoculum externe sur la propagation $\mathrm{du}$ CABMV (Cowpea aphid - borne mosaic virus) selon la proximité des parcelles voisines et la variété de niébé. Int. J. Biol. Chem. Sci., 9(5): 2309-2323.

Néya BJ, Zida PE, Sérémé D, Lund OS, Traoré O. 2015. Evaluation of Yield Losses Caused by Cowpea Aphid-borne mosaic virus (CABMV) in 21 Cowpea (Vigna unguiculata (L.) Walp.) Varieties in Burkina Faso. Pakistan J. Biol. Sci., 18(7): 304-313. DOI: 10.3923/pjbs.2015.304.313

Niang ISO. 2004. Cowpea productivity improvement In the African Agricultural Technology. Foundation (AATF).

NG JCK, Perry KL. 2004. Transmission of plant viruses by aphid vectors. Mol. Plant Pathol., 5: 503-511. DOI: 10.1111/j.1364-3703 .2004. 00240.x.
Ouédraogo S. 2003. Impact économique des variétés améliorées du niébé sur les revenus des exploitations agricoles du plateau central du Burkina Faso. Tropicultura, 21: 204-210.

Uganda Orawu M, Obuo J, Omadi R. 2015. Distribution and detection of cowpea viruses infecting cowpea. American Journal of Plant Sciences, 6: 574-581. DOI:

https://doi.org/10.4236/ajps.2015.65062

Sharma P, Kumar AS, Verma RK, Mishra R, Choudhary DK, Gaur RK, 2013. Biological and Molecular characterization of Cowpea aphid-borne mosaic virus causing severe mosaic disease in cowpea, India. Int. J. Advanced Research, 8: 744-749. http://www.journalijar.com

Wulandari DR, Ermayanti TM. 2011. Detection of potyvirus using RT-PCR and ACP-ELISA of Dioscorea species and in vitro shoot multiplication of the virus free plants. Annales Bogorienses, 15(2): $1-8$.

USDA. 2004. USDA national nutrient database for standard reference, release 17. (Internet) U.S. Department of Agriculture, Agricultural Research Service, Nutrient Data laboratory, Beltsville Md, United States. USDA. http://www.nal.usda.gov/fnic/foodcomp. 